\title{
EL PEI COMO SOPORTE IDEOLÓGICO DE LA REVOLUCIÓN EDUCATIVA ECUATORIANA
}

\author{
PEI AS IDEOLOGICAL SUPPORT OF THE \\ ECUADORIAN EDUCATIONAL REVOLUTION
}

\author{
Alfonso Efraín Pesantes Martínez \\ Centro de Interpretación Pedagógica, Guayaquil, Ecuador. info@educar.ec
}

\section{RESUMEN}

El Proyecto Educativo Institucional, es un documento público de planificación estratégica, que tiene una duración de 4 años y de construcción permanente, parte desde un punto en el tiempo, comienza con la autoevaluación y analiza 5 campos de gestión, la administrativa, pedagógica, convivencia, servicios educativos y sistema integral de gestión de riesgos escolares. Posee tres campos que determinan la identidad institucional, la visión, misión e ideario. Identificamos el problema por el cual realizamos está investigación, los sistemas educativos están en crisis recurrentes y necesitan transformarse permanentemente, lo hacen desde los niveles centrales, pero existe el documento público relacionante entre el nivel superior y el centro educativo, el PEl, que necesita impregnarse con los elementos capaces de transformar la educación. Usando la metodología descriptiva analizamos la visión, misión e ideario de 14 centros educativos, llegamos a concluir que estos se construyen considerando tres enfoques predominantes: interno, externo y el combinado interno-externo. $Y$ es necesario, la construcción de la visión, misión e ideario con equilibrio ubicando y desarrollando las ideas fuerza para cada uno de los elementos siguientes: docente, estudiante, modelo, valor y el estándar. Terminamos con la presentación de una propuesta básica no exhaustiva, como herramienta para la construcción del PEl como soporte de la revolución educativa ecuatoriana. La planificación estratégica no es exclusiva de los centros educativos, porque a través del Plan Plurianual de Políticas Públicas (PPPP) y el Plan de Desarrollo Institucional (PEDI), las instituciones del Nivel de Educación Superior articulan sus avances y alcances.

Palabras claves: Proyecto Educativo Institucional, Visión, Misión, Ideario

\begin{abstract}
The Institutional Educational Project, is a public document of strategic planning, which lasts for 4 years and is permanently built, it parts from a point in time, begins with the self-assessment and analyzes 5 fields of management, administrative, pedagogical, coexistence, educational services and comprehensive school risk management system. It has three fields that determine the institutional identity, vision, mission and ideology. The authors identified the problem, for that reason this research was carried out, the educational systems are in recurrent crisis and need to be permanently transformed, this is made from the central levels, but there is a public document relating between the upper level and the educational center, the PEI, which needs to be impregnated with the elements capable of transforming education. The descriptive methodology allowed to analyze the vision, mission and ideology of 14 educational centers, it was concluded that these are built considering three predominant approaches: internal, external and the internal-external combined. And it is necessary, the construction of the vision, mission and ideology with balance locating and developing the ideas strength for each of the following elements: teacher, student, model, value and the standard. This study finishes with the presentation of a non-exhaustive basic proposal, as a tool for the construction of the PEI supporting the Ecuadorian educational revolution. Strategic planning is not exclusive of the educational centers, because through the Pluriannual Plan of Public Policies (PPPP) and the Plan of Institutional Development (PEDI), the institutions of the Level of Higher Education articulate their advances and scopes.
\end{abstract}

Keywords: Institutional Educational Project, Vision, Mission, Ideology

Recibido: 22 de febrero de 2018; Aceptado: 12 de abril de 2018; Publicado: 20 de junio de 2018 


\section{Introducción}

EI PEI según (Correa, 2012) cuando expidió la reglamentación de la $\mathrm{LOEI}$, dejó claro en su artículo 88, ' el Proyecto Educativo Institucional, es el documento público de planificación estratégica, e introduce los conceptos, vinculación propositiva y características diferenciadoras institucionales. Por otra parte, apoyado en (Bravo, 2016) El PEI para convertirse en documento público necesita ser suscrito por el directivo del centro educativo y sigue una secuencia de rituales, solemnidades y procesos, tomaréis en cuenta que no está excepta de la falsedad ideológica, siguiendo, el PEI debe aprobarse siguiendo las normativas de la Autoridad Educativa Nacional y, por último, registrarlo en el Distrito. El PEI, es el instrumento que orienta los 5 procesos de gestión que desarrollan los centros educativos propuestos por las matrices correspondientes. (Ministerio de Educación Ecuador, 2016) que son: gestión administrativa, pedagógica, convivencia, servicios educativos y sistema integral de gestión de riesgos escolares.

EI PEl desde una visión más amplia no debe sustraerse a la idea de ser proyecto históricosocio-cultural y de desarrollo institucional, asume posturas perspectivas y prospectivas desde la visión generalista que defiende la sociedad incluida en la constitución del Ecuador, considera en su construcción, la visión de sociedad que aspira el centro educativo, constituye el compromiso originado en las propias concepciones del educador que, consensuadas con la participación logran, los acuerdos mínimos orientadores de todos los procesos y prácticas del centro educativo. (Representación de la UNESCO en Perú, 2011) Defendemos la idea que, al crear, la visión, misión, valores e idearios, además matizando las concepciones del modelo socioconstructivista que propugna el Ministerio de Educación desde la Propuesta Curricular 2016, será posible trasformar la educación. Estas generalidades de los planes estratégicos, como es el PEI, con énfasis en los centros educativos medios, el Plan Plurianual de Políticas Públicas (PPPP) el Plan de Desarrollo Institucional (PEDI), en los centros de educación superior requieren construirse, no como mero soporte legal o normativo, sino que debe estar inspirado en la persona, el estudiante como centro y motivo del quehacer formativo de la educación.

\section{Problema}

Los sistemas educativos al percatarse que sus desempeños están por debajo del promedio mundial o regional plantean, la necesidad de revolucionar su sistema educativo. Definimos la revolución como la modificación, reordenamiento, creación de nuevas características de los componentes del sistema escolar con la correspondiente implementación de los medios para su articulación, los óptimos resultados que alcancen nos llevan a la conclusión que, se vive y se siente la revolución educativa. Dejamos claro que los cambios en sí mismo no conducen a una revolución y, pueden en algunas veces producir efectos inversos, la involución. La revolución educativa consiste en sacar del estancamiento al sistema educativo. Está demostrado que las trasformaciones más efectivas se producen en el aula de clases en la efectiva relación profesor-estudiante.

Si el cuerpo humano goza de buena salud es porque sus sistemas y las células que las constituyen funcionan adecuadamente. Si comparamos, la afirmación anterior con el sistema educativo, la analogía permitirá afirmar, los centros educativos son unidades técnicos-administrativas con características comunes cuya urdimbre forma todo el sistema y debe responder a las finalidades por las que fue constituido. También debemos dejar establecido que el ser humano es un universo bio-psico-social que actúa en comunidad y los centros educativos son creaciones artificiales constituidas dentro de un espacio temperoespacial que influye y es influido por la sociedad. Ahora el PEI, Proyecto Educativo Institucional, es el documento clave que estructura y orienta a los centros educativos y es el elemento relacionante entre la estructura jerárquica superior que representa a la autoridad Educativa Nacional y la comunidad educativa a la que pertenece. El problema fundamental de la educación para constituir el PEI como soporte de la revolución educativa estriba en construirlo siguiendo las normativas de la autoridad educativa nacional e impregnar de manera dirigida, la visión, misión, valores, ideario. El éxito obtenido por el centro educativo será la demostración inequívoca de la revolución educativa producida por el centro educativo. Si producimos la excelencia en un solo centro educativo, y los demás adoptan la metodología, cual juego de dominó, los demás centros educativos de los Circuitos Educativos, Distritos y Zona Escolar alcanzarán éxitos también terminará con, la modificatoria del sistema. Salta la pregunta ¿Cómo transformamos al PEI en elemento esencial y soporte para la revolución educativa ecuatoriana? ¿Cómo adquiere funciones transformadoras el Plan Plurianual de Políticas Públicas (PPPP) y el Plan de Desarrollo Institucional (PEDI), en la educación superior? entonces será necesario impregnarlos con 
soportes conceptuales que lleguen al estudiante preferentemente.

\section{Objetivos}

\section{General:}

Construir la propuesta inicial del proyecto educativo institucional a través de la personificación innovadora de los elementos directamente personifícales del PEI como son, la visión, misión, ideario y valores, para potencializarlo como instrumento de transformación, innovación y cambio de la educación ecuatoriana.

\section{Específicos}

- Determinar la filosofía educativa dentro del PEI

- Establecer la conexión educativa del PEI con el modelo socio-constructivista.

- Elaborar la visión, misión, idearios y valores del PEI con la innovación educativa.

\section{Supuestos}

EI PEI está construido a partir de una matriz generalista mecanicista, proporcionada por el ministerio, que no incluyó, el modelo educativo, no está articulado con alguna filosofía educativa consensuada que lo determine y lo fundamente.

EI PEI no está considerado como eje orientador de la trasformación educativa. La construcción participativa pasa por alto la profundidad de los componentes ideológicos que fundamentan los cambios de actitudes que, contribuyan al cambio social desde la educación de los sectores desfavorecidos de la sociedad.

Los sistemas educativos de todos los países aspiran alcanzar los mejores logros para su población.

Si es posible construir una matriz conceptual que evite trascripciones directas no fundamentadas ni consensuadas de los elementos ideológicos del PEI.

\section{Marco teórico}

Revisemos algo sobre el término. "Revolución. Del lat. tardío revoloteo, -ōnis. -en su opción- 4. f. Cambio rápido y profundo en cualquier cosa." (RAE, 2017). La revolución educativa, será entonces el cambio rápido y profundo que sufre el sistema hacia el logro de estándares establecidos. Ahora si definimos la educación como la acción de educar, inmediatamente nos encontramos con el problema sobre las finalidades de la educación, que al construirlo conforma los elementos del sistema. En algunos momentos, los centros y los sistemas educativos plantean la posibilidad de la revolución educativa.

\section{El caso Colombia}

Colombia ya en el periodo 2002-2006 plantea la necesidad de hacer una revolución educativa basada en los mínimos rendimientos de su sistema escolar y su baja cobertura, quiere revertir la tendencia, aumentar la calidad y elevar la cobertura. Las líneas macro de su plan de desarrollo educativo son tres políticas "1.Ampliar la cobertura educativa. 2.- Mejorar la calidad de la educación. 3.- Mejorar la eficiencia del sector educativo" (Ministerio de Educación Nacional, 2003) Existen otros elementos no menos importantes como la investigación científica y mejorar la educación superior, donde el estado pone su mejor esfuerzo.

\section{El caso argentino}

El eje de la revolución Argentina es la escuela porque allí se "...gesta el futuro del futuro (Consejo Federal de Educación, pág. 1)". Valora el aprendizaje continuo, favorece la evaluación. Eleva al maestro como autoridad educativa $y$, agente de cambio cultural. El apoyo de la sociedad es fundamental. Considera los principios como ejes de la revolución educativa y los declara y sin dejar de establecer la agenda de cambio. Invito a leer la Declaración de Purmamarca (Consejo Federal de Educación, 2016).

\section{Avance de los sistemas educativos del mundo}

Para la intervención siempre será necesario tomar en cuenta desde que punto parte la trasformación de los sistemas. En segundo lugar, determinar las intervenciones necesarias en los elementos del sistema a cambiar y, en tercer lugar, monitorear como el sistema se adapta a los cambios según su contexto.

Interesante agregar la sustentabilidad y la puesta en marcha, esos elementos colocan a los sistemas en el camino de las mejoras, independientemente de su ubicación con relación a la calidad. Algunos sistemas mejoran más rápidos que otros y conocer como lo hacen es la clave. La mejora produce un fenómeno denominado "mecanismos del sistema", la calidad y profundidad de las intervenciones consiguen los éxitos esperados. (Mourshed, Chijioke, \& Barber, 2012) para conseguir la 
revolución educativa un grupo de docentes desde la dirección de la educación o desde la base movilizar el sistema con intervenciones oportunas y efectivas. Las políticas públicas deben movilizar al sistema desde el nivel macro hasta el nivel micro del aula. Afirmamos que el punto ideal para planificar intervenciones sería el PEI por constituir el documento orientador de la sinergia de los centros educativos. En nuestro caso, el estudio está centrado y pone énfasis en los centros de educación media, no descartamos, las necesidades de impregnar a los centros de educación superior a través del PPPP y del PEDI, los elementos relevantes que consideren los colectivos académicos para la transformación educativa.

\section{El Proyecto Educativo Institucional, PEI}

EI PEI, por el lado del Reglamento de la LOEI, Art. - 88, es el "...documento público de planificación estratégica..." y por el lado del instructivo de la Actualización de la Guía Metodológica del PEI valora, el concepto de gestión como orientador de procesos internos vinculado al aprendizaje y su mejora. (Ministerio de Educación Ecuador, 2016) Dejemos claro, la excelencia de los aprendizajes alcanzado por los estudiantes son el producto de la gestión completa del centro educativo.

La estructuración del PEI, dentro del sistema educativo ecuatoriano se encuentra direccionado a través de los anexos 1,2 y 3 , proporcionados por la guía metodológica, dejando, el espacio indispensable para dentro del trabajo participativo direccionar, la visión, misión, ideario y valores.

El esquema básico del PEI es el siguiente:

- Índice

- Introducción

- Visión

- Misión

- Ideario

- Principios.

- Valores.

- Política General.

- Informe de la Autoevaluación Institucional

- Plan/es de mejora

- Evaluación y monitoreo

- Propuestas de innovación curricular.

- Programa de Participación Estudiantil

- Código de convivencia

Es indispensable dejar aclarado, la construcción del PEI, debe seguir las normas de la actualización curricular (Ministerio de Educación Ecuador, 2016)

\section{EI PEl integrador de recursos humanos}

El centro educativo es una organización social con objetivos predeterminados que actúan en el ambiente social, construyéndose y reconstruyéndose, así como se definen y redefinen sus objetivos. Ya decía (Chiavenato, 2011) que las organizaciones, para nosotros la escuela, recibe insumos de entrada, como sería las leyes, reglamentos y, orientaciones externas e internas, que luego son procesadas por los sistemas y subsistemas internos, como sería el centro educativo, la administración educativa y su consecuente, la acción pedagógica directa que, será la relación estudiante-profesor y, como todo proceso tiene su salida en los centros educativos será cumplir con los estándares educativos. Toda salida merece ser evaluada para producir la realimentación que es la acción que se ejerce sobre la entrada para reorientar el proceso y conseguir la salida esperada mejorada. En los centros educativos será cumplir con la finalidad para los que fueron creados. Las instituciones educativas son sistemas bien estructurados. Pero no debemos olvidar que según el enfoque de Tavistock, comentado por (Chiavenato, 2011), las instituciones son la combinación de tecnología y personas en interrelación recíproca. En definitiva, el sistema educativo de cualquier nivel concentra su atención a los mecanismos de entrada, el proceso y la salida. Graficándolo, será como un tubo o un embudo. El tubo, entra un grupo de estudiantes y son los mismos los que salen. En el caso del embudo, entran muchos y salen pocos. El otro momento será la preocupación, lo cualitativo, ¿Cuál es la calidad de los egresados? Todo depende del proceso, el CEAACES, ha respondido con la categorización de las universidades, además del esfuerzo propio de cada universidad con su proceso de autoevaluación. Evaluar las salidas profundamente con procesos integradores, no discriminatorios son los estudios pendientes.

La manera más eficaz de conciliar las finalidades de la organización y las personas es estructurar la Misión Organizacional que, es la declaración consiente de propósitos, su razón de ser y el diseño de como satisfacer las necesidades externas, debe conciliar con el ambiente y las personas para definir la escala de valores relacionándolo a nivel de las creencias de cómo desarrollar las acciones encomendadas con el menor esfuerzo posible.

La visión, como declaración inspiradora, también deberá conciliar los objetivos generales de las finalidades de los centros educativos en su mirada a largo plazo con, los intereses de los docentes. Debe generar un 
enfoque que partiendo de la cotidianidad signifique que cada día de labor, los esfuerzos combinados labren el futuro anhelado, solo posible lograrlo si lo construimos desde el presente.

El ideario, principios, valores y la política general constituyen elementos que vinculados tanto a la persona como al centro educativo conducen las acciones desde ángulos de las convicciones personales en concordancia con los objetivos institucionales. Los objetivos institucionales conforman el elemento fundamental del plan del centro educativo y obedece a los propósitos del proyecto institucional y requiere, la entrada de insumos para su trasformación y adecuada salida. Manejar estos elementos en la dirección de la trasformación educativa será la adicción de los docentes que quieran hacer la revolución educativa, no importa de qué espacio partimos, si somos reglares, buenos o muy buenos. La articulación de los componentes filosóficos y educativos dará, la certidumbre a los anhelos personales e institucionales como los de la sociedad. $Y$ si queremos revolución, los elementos ideológicos deben construir, modificar, difundir e implementar cambios que partan del centro educativo hacia la comunidad educativa.

\section{Planteamiento y justificación}

La revolución educativa necesita la articulación de los elementos ideológicos del PEI ( Visión, Misión, Ideario ...) con el proyecto general del centro educativo y según (Pardilla, 2014) una ideología unificadora que también considere las aspiraciones de la comunidad educativa y fusione en un todo, los anhelos de la sociedad con relación al centro educativo y porque no también, las ciencias y tecnologías de la educación, evitar eso sí que, las marcas ideológicas trasciendan hacia la periferia, de la unidad distrital que pertenece el centro educativo, la idea no es tornar el centro educativo en una central política, aunque algunos distritos educativos, muchas veces, pudieren eventualmente funcionar como tal como, la evidencia fotográfica lo demuestra.

Usaré la metodología descriptiva. Como estamos analizando la visión, misión, ideario del PEI, arbitrariamente usaré una muestra de 14 PElii, de 14 centros educativos para analizar las características esenciales a partir de clasificarlos según sus orientaciones generales con relación a sus enfoques y centralidades más pronunciada que son: interno, externo 0 combinado, es decir, interno-externo. Todos estos enfoques están en correspondencia con el centro educativo, la comunidad, la ideología y los fundamentos de la pedagogía.

Enfoque Interno. - La construcción se refiere a crear condiciones adecuadas para la acción dentro de la comunidad educativa sin considerar o poco considerar las condiciones o influencias sociales externas donde está inmersa el Centro Educativo.

Enfoque Externo. - La construcción se refiere a crear condiciones adecuadas para que la acción educativa pueda constatarse, observarse y ofrecerse para la comunidad educativa, sin considerar o poco considerar las condiciones o influencias sociales internas donde está inmersa el Centro Educativo.

Enfoque Interno - externo. - La construcción de la visión, misión e ideario considera las acciones para ser constatadas tanto desde lo interno de las instituciones educativas como desde lo externo de las mismas. Es la combinación de los aspectos internos como externos de las instituciones educativas en equilibrio.

Análisis de la visión. - Analizados los 14 PEI, constatamos que, la visión está enfocada con mayor magnitud hacia situaciones internaexterna. Al observar el Cuadro \# 1 encontramos que el efecto combinado, interno - externa, alcanza 9 casos. Para la visión interna, 4 casos, el enfoque externo 1 caso.

\section{Cuadro \# 1}

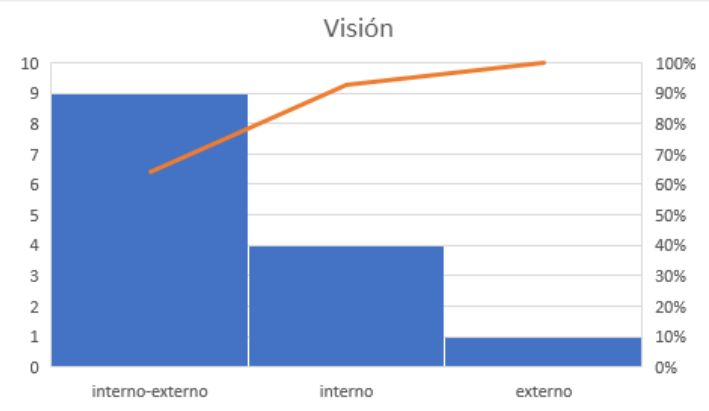

Análisis de la Misión. - La Misión tiene equilibrio. El cuadro \# 2 encontramos que el enfoque interno alcanza 7 casos, el enfoque de la misión interna-externa 7 casos y para la visión externa 0 caso. Existe un equilibrio entre lo interno y lo externo. 


\section{Cuadro \# 2}

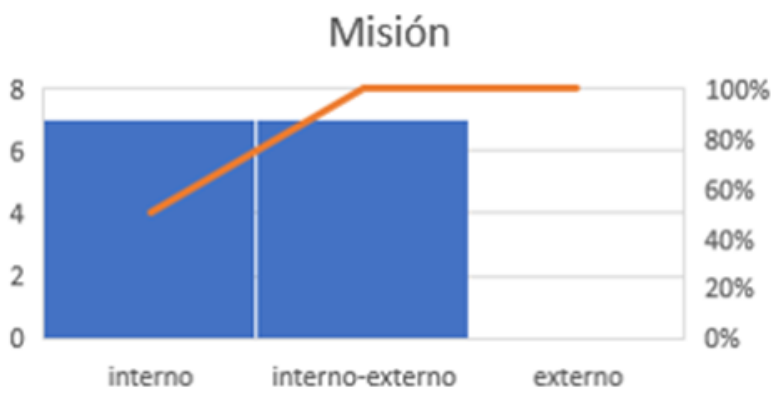

Análisis del Ideario. - El enfoque externo predomina alcanza 8 casos, los enfoques interno-externo 5 casos y para el efecto interno 0 casos. Cuadro \# 3 .

\section{Cuadro \#3}

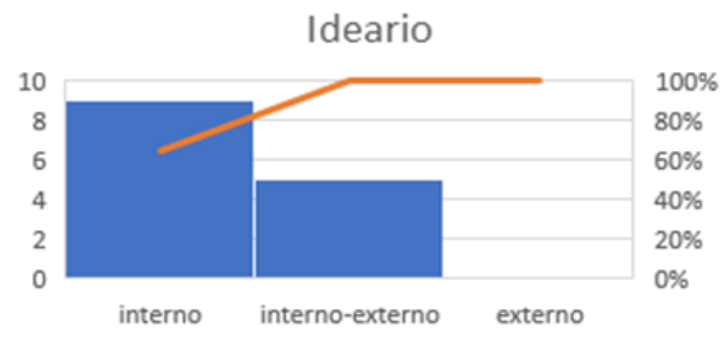

Análisis global. - Ahora los datos presentados en el cuadro \# 4, la Visión, Misión e Ideario. El enfoque interno- externo, alcanza el $50 \%$, y el interno posee el $48 \%$, técnicamente un equilibrio. Unificando los enfoques internos y externos alcanza el $98 \%$ que representa la mayoría, cerca de la totalidad.

\section{Cuadro \# 4}

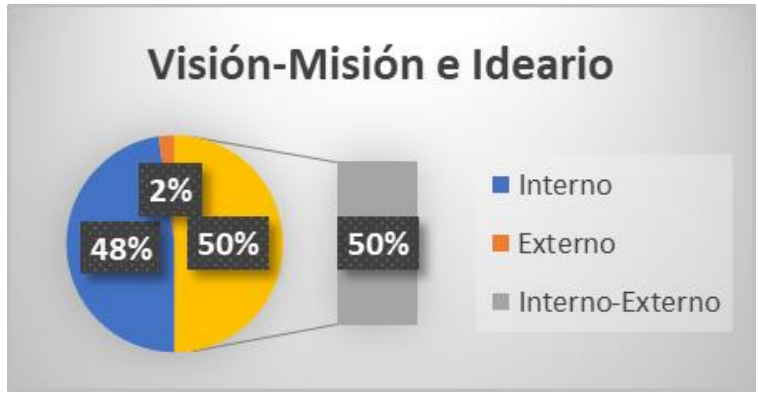

Comentario General. - La visión, misión, e ideario son construcciones personalizadas para encontrar la identidad institucional, Si bien los enfoques pueden estar al servicio interno, externo o combinando. Jamás podremos decir que están incorrectos debido a que, si las ideas fuerza son lo suficientemente potentes, el cambio, transformación, innovación se hará presente por la misma fuerza de la convicción, la aplicación de las ciencias de la educación y uso de las tecnologías de la información. Pero esto tampoco significa renunciar la posibilidad de crear una propuesta matriz que apoye este proceso según la direccionalidad que le puede dar los centros educativos. En nuestro caso será convertir el PEI en soporte de la revolución educativa. Visite en la Zona 8, Distrito Ximena 1. Dos centros educativos, al requerir sobre el $\mathrm{PEI}$, dijeron que el documento, "Actualización de la guía metodológica para la construcción participativa del Proyecto Educativo Institucional," (2016), lo están trabajando para actualizar el PEI. En cuanto al registro del PEI, (Art. 88 del Reglamento de la LOEI) en el Distrito Ximena 1. Solo han ingresado 15 PEI sin los nuevos códigos, ahora están ajustando, el sistema informático para ordenar que las instituciones los envíen para el registro. En cuanto a la Auditoría del PEI, por parte de los Auditores, art 88 del reglamento al 11/4/2018, no lo ha sido realizada con la nueva actualización. El universo del Distrito Ximena 1, está constituido por 100 planteles públicos y 80 particulares, expresó el Máster Rafael Martillo Merchán, que debidamente informado del proceso investigativo actuó como vocero del Distrito Ximena 1.

\section{La propuesta}

Planteamos el equilibrio dinámico y articulado de la visión, misión e ideario, integrando, las declaraciones y las ideas fuerza, considerando los factores internos-externos tal como lo expresa el cuadro \# 5. Los aspectos internos recogen las aspiraciones centrales del centro educativo sobre el desarrollo conceptual, tecnológico, pedagógico y, por último, visualiza - presupone la posición ontológica, antropológica, sociológica, axiológica, epistemológica y pedagógica.

Los factores externos corresponden a cómo queremos que nos observen los demás centros educativos, esto es involucrarse con la comunidad, desarrollando la dimensión externa, estableciendo compromisos ante la comunidad, con la comunidad y para la comunidad, creando lazos de responsabilidad. Crear medios para verificar el seguimiento a través de la rendición de cuentas y conocer el grado de alcance desde, lo que tenemos hasta lo que queremos. 


\section{Cuadro \# 5}

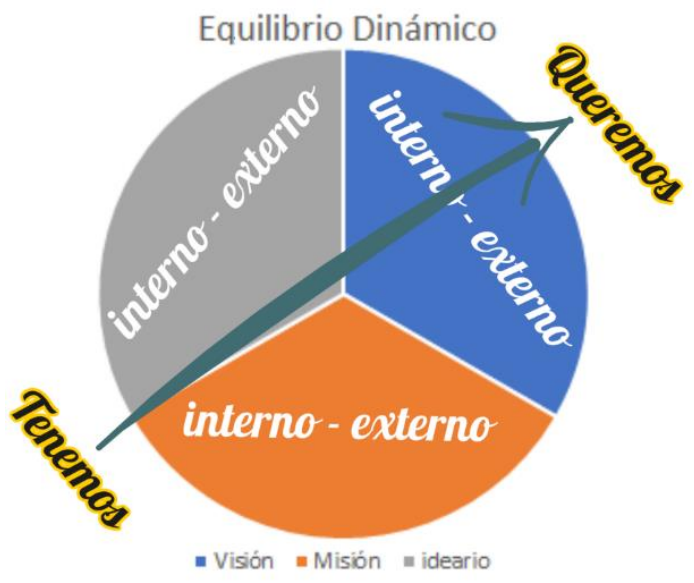

La construcción de la visión, misión e ideario

Para la construcción de la visión, misión e ideario debemos proyectarnos, reiterando desde lo que tenemos hasta lo que queremos, considerando a la persona: estudiante y docente, así como incluir al modelo educativo por su riqueza conceptual subyacente, afianzar el valor eje a partir del cual construir los demás valores y, hacer referencia al estándar porque es, la guía perspectivas y prospectiva de la calidad educativa.

\section{Cuadro \# 6}

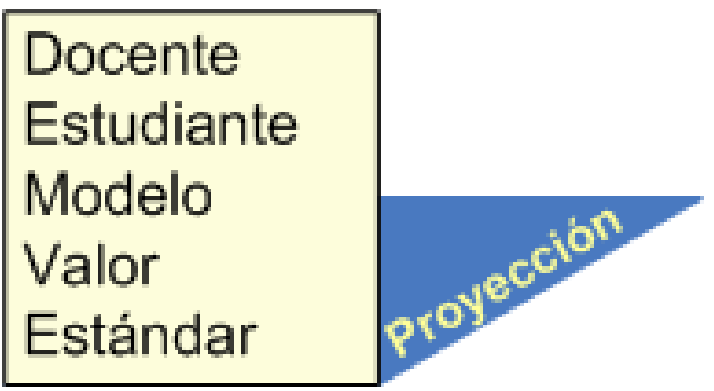

Propuestas de declaración

Visión (acercamiento conceptual)

Alcanzaremos la excelencia a base del esfuerzo sostenido del docente y la superación constante del estudiante, aportaremos durante cada periodo anual al desarrollo del modelo social constructivista, la responsabilidad será el eje para la adopción paulatina de las mejores actitudes humanas para estar en posibilidad de crecer constantemente y alcanzar los mejores niveles de la evaluación según los estándares nacionales.

Comentario: Esta visión está construida siguiendo el eje del crecimiento permanente comenzando del punto $A$ hacia el punto $B$, es decir desde lo que tenemos hasta lo que queremos.

\section{Misión (acercamiento conceptual)}

Conformarnos en comunidad articulada de aprendizaje desde cada posición a la que pertenecemos, docentes, estudiantes y comunidad; educamos desde la vocación para la integración social; impulsamos desde la práctica, el modelo socio constructiva de la educación; nos proponemos, la educación en valores $y$, desde el desarrollo curricular estamos enfocados en que los estudiantes alcancen los estándares de la educación.

Comentario: Bueno, la idea es constituirse en comunidad articulada de aprendizaje y, como tal proyectar al interior del centro educativo para que, el educando fundamente su acción desde la pedagogía de la construcción de conocimiento con el apoyo docente.

\section{Cuadro \# 7}

Docente y estudiante
(¿Quiénes somos?)
Modelo ¿ Por qué y para
que educamos? ¿Qué y
¿cómo? lo hacemos?
Valor ¿Qué nos
proponemos?
Estándar ¿ A quién
educamos?

Ideario (acercamiento conceptual)

Somos trabajadores de la educación, ubicamos al estudiante como centro del esfuerzo educativo, practicamos para enseñar, los valores y derechos humanos, la democracia, la justicia, solidaridad y planteamos el hombre nuevo con responsabilidad social que domine la tecnología.

Somos docentes despiertos al crecimiento personal desde la investigación como medio de alcanzar la autonomía pedagógica para aplicar las orientaciones desde ámbitos prospectivos que permitan desde el presente construir el futuro.

Somos estudiantes abiertos a los cambios con disciplina y tenacidad para alcanzar los objetivos personales aprovechando la ubicación tempero-espacial que la vida nos ha favorecido, orientamos nuestra vida con el lema: Buscamos la verdad.

Desarrollaremos con convicción el modelo socio constructivista de la educación desde las 
orientaciones proporcionada por el poder político y la interpretación directa de sus postulados fundamentándolos con los datos que la realidad inmediata nos proporciona la comunidad educativa interna institucional y la comunitaria durante la indagación científica.

Estamos convencidos que practicando el valor de la responsabilidad será el fundamento para incorporar los demás valores esenciales para la convivencia social y el desarrollo de las ciencias.

\section{Conclusiones}

La idea de cambio, revolución, innovación, trasformación constituye una constante dentro de todos los sistemas educativos.

EI PEI con sus componentes ideológicos, la visión, misión e ideario son soportes esenciales para impregnar las ideas fuerzas capaces de vencer la resistencia psicológica al cambio.

Del análisis de los PEI deducimos la necesidad de construir los renovados PEI con equilibrio y articulación entre los elementos impregnables durante la construcción participativa.

La visión, misión e ideario deben construirse considerando la comunidad de aprendizaje interna y la comunidad externa en lo que hemos denominado enfoque interno-externo, los postulados deben ser equilibrada y articulada considerando, al docente, el estudiante, el modelo, el valor y el estándar.

La propuesta presentada es muy básica y deben concebirse desde una metodología inductiva-deductiva en sendos talleres de construcción conceptual.

EI PEI, PPPP. PEDI, son documentos normativos que deben ser auditados de manera permanente por todas las instancias que correspondan, mantenerlos los planes en constante construcción, sin desviarse del objetivo central de la educación, que es formar personas dándole las necesarias competencias profesionales al egresar. Tomar cada uno de los niveles educativos en lo interno, como también de la educación misma que ofertan los centros educativos.

\section{Bibliografía}

Bravo, H. (2016), FALSEDAD DE
INSTRUMENTO PÚBLICO: DECLARATO-
RIA, CELERIDAD Y ECONOMÍA PROCE-
SAL. Guayaquil: UNIVERSIDAD REGIONAL AUTÓNOMA DE LOS ANDES "UNIANDES"/Universidad de Guayaquil. Obtenido de http://dspace. uniandes. edu.ec/bitstream/123456789/4291/1/TUAM DC004-2016.pdf

Chiavenato, I. (2011). Administración de Recursos Humanos. México: Mc Graw Hill Educación . Obtenido de https://www.upg. mx/wp-content/uploads/2015/10/LIBRO-12Administracion-de-recursos-humanos.-Elcapital-humano.pdf

Consejo Federal de Educación. (2016). Declaración de Purmamarca. (pág. 4). Jujuy: Consejo Federal de Educación. Obtenido de http://www.me.gov.ar/consejo /resoluciones/res16/purmamarca_16.pdf

Correa, R. (2012). REGLAMENTO GENERAL A LA LEY ORGÁNICA DE EDUCACIÓN INTERCULTURAL codificado. Quito, Ecuador: Ministerio de Educación. Obtenido de https://educacion.gob.ec/wp-content/up loads/downloads/2016/03/LOEI-Actualizado .pdf

Ministerio de Educación Ecuador. (2016). Actualización de la guía metodológica para la construcción participativa del Proyecto Educativo Institucional. Quito, Pichincha, Ecuador: Ministerio de Educación de Ecuador. Obtenido de https://educacion. gob.ec/wp-content/uploads/downloads/ 2017/03/Guia-Metodologica-para-laConstruccion-Participativa-del-ProyectoEducativo-Institucional.pdf

Ministerio de Educación Nacional. (1 de marzo de 2003). https://www.mineducacion. gov.co. Obtenido de https://www. mineducacion.gov.co/1621/articles-85266 archivo_pdf.pdf

Pardilla, S. (31 de 03 de 2014). http://ssociologos.com. Recuperado el 24 de 1 de 2018, de http://ssociologos.com: http://ssociologos.com/2014/03/31/element os-para-hacer-una-revolucion/

Representación de la UNESCO en Perú. (2011). Manual de Gestión para Directores de Instituciones Educativas. Lima: Lance Grafico S.A.C. Obtenido de http://unesdoc.unesco.org/images/0021/00 2191/219162s.pdf 
i CAPÍTULO V Reglamento de la LOEI

DEL PROYECTO EDUCATIVO INSTITUCIONAL

"Art. 88.- Proyecto Educativo Institucional. El Proyecto Educativo Institucional de un establecimiento educativo es el documento público de planificación estratégica institucional en el que constan acciones estratégicas a mediano y largo plazo, dirigidas a asegurar la calidad de los aprendizajes estudiantiles y una vinculación propositiva con el entorno escolar. El Proyecto Educativo Institucional debe explicitar las características diferenciadoras de la oferta educativa que marquen la identidad institucional de cada establecimiento. Se elabora de acuerdo con la normativa que expida el Nivel Central de la Autoridad Educativa Nacional, y no debe ser sometido a aprobación de instancias externas a cada institución; sin embargo, estas lo deben remitir al Nivel Distrital para su registro. En las instituciones públicas, el Proyecto Educativo Institucional se debe construir con la participación del Gobierno escolar; en las instituciones particulares y fiscomisionales, se debe construir con la participación de los promotores y las autoridades de los establecimientos. Las propuestas de innovación curricular que fueren incluidas en el Proyecto Educativo Institucional deben ser aprobadas por el Nivel Zonal. La Autoridad Educativa Nacional, a través de los auditores educativos, debe hacer la evaluación del cumplimiento del Proyecto Educativo Institucional."

ii Anexo (Visión, Misión, Ideario de cada plantel)

Fuente de cada PEI de los planteles

(1) http://colegioamericano.edu.ec/pei/

(2) http://www.ueprim.edu.ec/website/images/documentos/PEl2013-2017.pdf

(3) http://ang.edu.ec/quevedo/documentos/pdf/PEI 2017.pdf

(4) http://wwwfs.mineduc.cl/Archivos/infoescuelas/documentos/11394/ProyectoEducativo11394.pd $f$

(5) htttp://www.colegiobenignomalo.edu.ec/docs/peibm.pdf

(6) http://www.montessori.edu.ec/descargas/PEl.pdf

(7) $\mathrm{http://www.santamariadelosangeles.cl/www/doc/PEl-2016.pdf}$

(8) http://www.monografias.com/trabajos98/proyecto-educativo-escuela-miguel-riofrio-lojaecuador/proyecto-educativo-escuela-miguel-riofrio-loja-ecuador.shtml

(9) http://nuevedeoctubre.edu.ec/publico/documentos/PEl.pdf

(10) http://www.colegiodelapresentacion.edu.co/conte/nuestro colegio/PEl.pdf

(11)http://www.educacion.quito.gob.ec/unidades/sebastiandebenalcazar/images/contenidos/hide/2 015/Common/PEl.pdf

(12)http://itinelsantuario.edu.co/docs/PEl\%20JMC\%202015-2018.pdf

(13)https://sites.google.com/site/instedulainmaculada/pe

(14)https://www.medellin.gov.co/ir/go/km/docs/educacionNuevo01dic/iesoldeoriente/documentos/P EI\%20SOL\%20DE\%20ORIENTE\%202009.pdf 